\title{
Observation of Stimulated Brillouin Scattering in Silicon Nitride Integrated Waveguides
}

\author{
Flavien Gyger@, ${ }^{1, *}$ Junqiu Liu, ${ }^{2, *}$ Fan Yang $\odot,{ }^{1, *}$ Jijun $\mathrm{He}^{2},{ }^{2}$ Arslan S. Raja, ${ }^{2}$ Rui Ning Wang, ${ }^{2}$ \\ Sunil A. Bhave $\odot^{3}$ Tobias J. Kippenberg, ${ }^{2, \dagger}$ and Luc Thévenaz $\odot^{1, \hbar}$ \\ ${ }^{1}$ Group for Fibre Optics, Swiss Federal Institute of Technology Lausanne (EPFL), CH-1015 Lausanne, Switzerland \\ ${ }^{2}$ Laboratory of Photonics and Quantum Measurements, Swiss Federal Institute of Technology Lausanne (EPFL), \\ CH-1015 Lausanne, Switzerland \\ ${ }^{3}$ OxideMEMS Lab, Purdue University, 47907 West Lafayette, Indiana, USA
}

(Received 10 September 2019; published 3 January 2020)

\begin{abstract}
Silicon nitride $\left(\mathrm{Si}_{3} \mathrm{~N}_{4}\right)$ has emerged as a promising material for integrated nonlinear photonics and has been used for broadband soliton microcombs and low-pulse-energy supercontinuum generation. Therefore, understanding all nonlinear optical properties of $\mathrm{Si}_{3} \mathrm{~N}_{4}$ is important. So far, only stimulated Brillouin scattering (SBS) has not yet been reported. Here we observe, for the first time, backward SBS in fully cladded $\mathrm{Si}_{3} \mathrm{~N}_{4}$ waveguides. The Brillouin gain spectrum exhibits an unusual multipeak structure resulting from hybridization with high-overtone bulk acoustic resonances of the silica cladding. The reported intrinsic $\mathrm{Si}_{3} \mathrm{~N}_{4}$ Brillouin gain at $25 \mathrm{GHz}$ is estimated as $4 \times 10^{-13} \mathrm{~m} / \mathrm{W}$. Moreover, the magnitude of the $\mathrm{Si}_{3} \mathrm{~N}_{4}$ photoelastic constant is estimated as $\left|p_{12}\right|=0.047 \pm 0.004$, which is nearly 6 times smaller than for silica. Since SBS imposes an optical power limitation for waveguides, our results explain the capability of $\mathrm{Si}_{3} \mathrm{~N}_{4}$ to handle high optical power, central for integrated nonlinear photonics.
\end{abstract}

DOI: 10.1103/PhysRevLett.124.013902

Introduction.-Amorphous silicon nitride $\left(\mathrm{Si}_{3} \mathrm{~N}_{4}\right)$ [1] shows exceptional performance in terms of low linear optical losses below $1 \mathrm{~dB} / \mathrm{m}$ and the absence of twophoton absorption, and has been widely used for passive elements [2]. In addition, the high Kerr nonlinearity and flexibility to engineer the anomalous group velocity dispersion (GVD) [3] have made $\mathrm{Si}_{3} \mathrm{~N}_{4}$ an ideal platform for integrated nonlinear photonics. Recent advancements in nanofabrication have overcome the highly tensile film stress of stoichiometric $\mathrm{Si}_{3} \mathrm{~N}_{4}$ [4,5]. These developments allow high-yield fabrication of $\mathrm{Si}_{3} \mathrm{~N}_{4}$ waveguides with tight optical confinement and anomalous GVD, as required for parametric frequency conversion via Kerr nonlinearity [6]. Such integrated $\mathrm{Si}_{3} \mathrm{~N}_{4}$ waveguides are presently a leading platform for soliton microcomb generation [7]. Integrated $\mathrm{Si}_{3} \mathrm{~N}_{4}$-based soliton microcombs can now operate with ultralow electrical driving power [8,9] and repetition rates extending down into the microwave domain [10]. In addition, $\mathrm{Si}_{3} \mathrm{~N}_{4}$ waveguides enable coherent and lowpulse-energy supercontinuum generation in the near infrared [11], as well as the midinfrared for dual-comb spectroscopy [12]. Other nonlinear phenomena in $\mathrm{Si}_{3} \mathrm{~N}_{4}$ waveguides, such as second- and third-harmonic generation $[13,14]$, as well as stimulated Raman scattering [15] have

Published by the American Physical Society under the terms of the Creative Commons Attribution 4.0 International license. Further distribution of this work must maintain attribution to the author(s) and the published article's title, journal citation, and DOI. been studied. However, to date, there is no report on backward Brillouin scattering in $\mathrm{Si}_{3} \mathrm{~N}_{4}$.

Stimulated Brillouin scattering (SBS) is a nonlinear process mediated by an acousto-optic interaction inside a medium [16-18]. It has been observed in various platforms, including silica fibers [19-23], whispering-gallery-mode resonators [24-27], and integrated waveguides based on chalcogenide [28,29], silicon [30,31] and aluminum nitride [32] (electromechanically induced). SBS has led to several applications, such as slow and fast light [33,34], microwave photonic filters [30,35], microwave synthesis [36], highly coherent laser sources [37,38], gyroscopes [38,39], isolators [40-42], mode-locked lasers [21,43] and sensors [44]. At the same time, Brillouin scattering poses a power limitation in waveguides and fibers [45], and in addition induces noises via thermal excitation of guided acoustic waves.

Recently, integrated $\mathrm{Si}_{3} \mathrm{~N}_{4}$ waveguides have been used to demonstrate a Brillouin laser [38]. However, the SBS interaction occurred within the silica $\left(\mathrm{SiO}_{2}\right)$ cladding, as confirmed by the Brillouin frequency shift of $10.9 \mathrm{GHz}$. A second work proposed the use of a $\mathrm{Si}_{3} \mathrm{~N}_{4}$ membrane including a waveguide and a phononic crystal to explore forward SBS $[46,47]$. A third work demonstrated a large light-sound interaction using forward SBS in integrated silicon waveguides [30], while a $\mathrm{Si}_{3} \mathrm{~N}_{4}$ membrane was used to guide the transverse phonons. However, to date, no work has shown backward SBS in $\mathrm{Si}_{3} \mathrm{~N}_{4}$ material. Moreover, to the best of our knowledge, there is no reference to the $\mathrm{Si}_{3} \mathrm{~N}_{4}$ photoelastic tensor (e.g., $p_{11}$ and $p_{12}$ ) in the literature. 


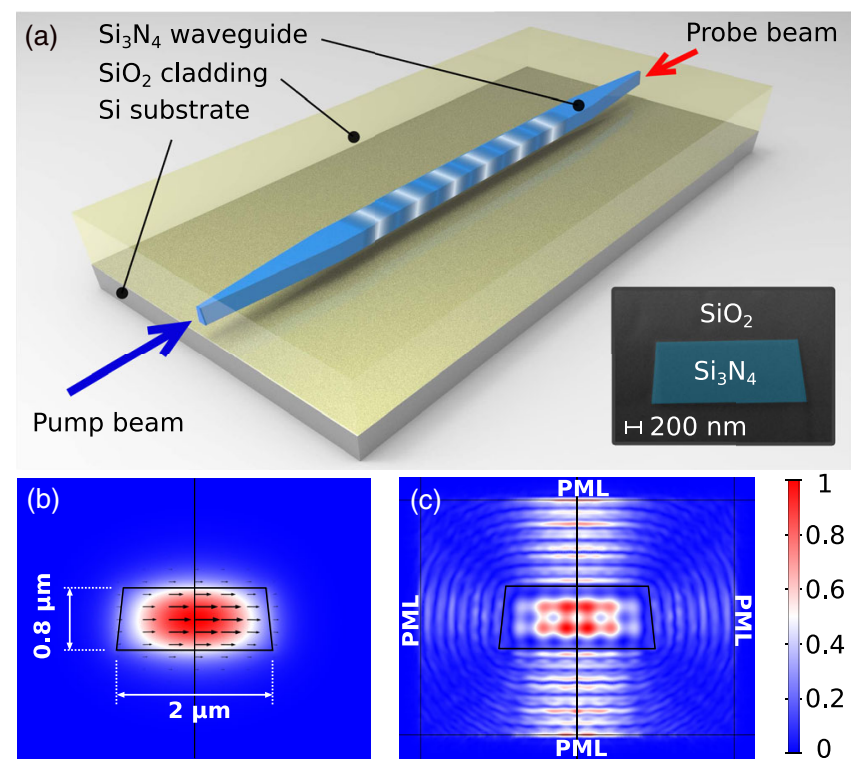

FIG. 1. On-chip SBS in a $\mathrm{Si}_{3} \mathrm{~N}_{4}$ waveguide. (a) Artist's view of $\mathrm{SBS}$ in a $\mathrm{Si}_{3} \mathrm{~N}_{4}$ waveguide. Note that the length of the inverse tapers is exaggerated for illustrative purpose. The actual taper length is $300 \mu \mathrm{m}$, while the full length of the $\mathrm{Si}_{3} \mathrm{~N}_{4}$ waveguide is $5 \mathrm{~mm}$. White and blue shading represents the material density variation generated by SBS when the pump and probe beams overlay. Inset: false-color SEM of the waveguide cross section of $2 \times 0.8 \mu \mathrm{m}^{2}$. (b) Normalized electric field distribution for the optical TE-like mode. The arrows represent the direction and strength of the electric field in the cross-section plane. (c) Normalized displacement field norm (instantaneous value) for the fundamental acoustic mode. The vertical black line visible in (b) and (c) is indicative of the waveguide symmetry.

In this Letter, we characterize, for the first time, the $\mathrm{Si}_{3} \mathrm{~N}_{4}$ backward SBS gain spectrum in a 5-mm-long waveguide buried in $\mathrm{SiO}_{2}$. Figure 1(a) shows an artist's view of SBS in a $\mathrm{Si}_{3} \mathrm{~N}_{4}$ waveguide buried in $\mathrm{SiO}_{2}$. Two optical fields, pump and probe, spatially overlap and are phase matched with an acoustic mode, which induces acoustic oscillations through electrostriction and subsequent generation of a moving grating. Reciprocally, acoustic waves scatter light via photoelasticity. The phase-matching condition is given by $\nu_{B}=2 n_{\text {eff }} v_{a} / \lambda$, where $n_{\text {eff }}$ is the effective refractive index of the optical mode, $\lambda$ is the probe wavelength, $v_{a}$ is the acoustic mode velocity and $\nu_{B}$ is the frequency detuning between the interacting light waves, called the Brillouin frequency shift. In the present case, our waveguide cross section is a $2 \times 0.8 \mu \mathrm{m}^{2}$ trapezoid, exhibiting a smaller width at the top [48], as shown in the false-color scanning electron microscopy (SEM) image in Fig. 1(a) inset. The refractive indices of $\mathrm{Si}_{3} \mathrm{~N}_{4}$ and $\mathrm{SiO}_{2}$ at $1550 \mathrm{~nm}$ are $n_{\mathrm{Si}_{3} \mathrm{~N}_{4}}=2.00$ and $n_{\mathrm{SiO}_{2}}=1.45$, respectively.

The waveguide fundamental quasitransverse electric (TE-like) mode is adiabatically excited by inverse nanotapers $[52,60]$ placed at chip facets. The simulated optical mode profile in the waveguide using the finite-element method (FEM) is shown in Fig. 1(b). The computed effective refractive index and mode area are $n_{\text {eff }}=1.85$ and $A_{\text {eff }}=1.2 \mu \mathrm{m}^{2}$, respectively. A solid mechanics simulation of the fundamental acoustic mode displacement field norm (instantaneous value) [48] is shown in Fig. 1(c). Acoustic waves propagate away from the waveguide and are absorbed by the surrounding perfectly matched layer (PML) due to the weak confinement caused by the lower acoustic velocity in $\mathrm{SiO}_{2}$ and by the small waveguide dimension [16,61]. The resulting eigenfrequency of this fundamental mode is $25.2+0.4 \mathrm{i} \mathrm{GHz}$, from which the imaginary part accounts for the phonon leakage rate. Moreover, the mode spatial distribution in the waveguide substantially deviates from the usual bell shape. This is caused by the small waveguide dimensions inducing hybrid acoustic modes [62]. Because of the negligible optical evanescent field in $\mathrm{SiO}_{2}$ cladding, SBS mainly takes place in $\mathrm{Si}_{3} \mathrm{~N}_{4}$. By using the measured photoelastic constant magnitude given in Eq. (3), our simulations confirm that the $\mathrm{SiO}_{2}$ contributes to $0.2 \%$ of the total Brillouin gain of the aforementioned acoustic mode.

Experiments and results. - Residual reflections between the two chip facets make the waveguide a low-finesse Fabry-Pérot (FP) cavity. The transmission spectrum of the waveguide is shown in Fig. 2(a), exhibiting a FP cavity free spectral range (FSR) of $\nu_{\mathrm{FSR}}=14.375 \mathrm{GHz}$, exactly corresponding to the $5-\mathrm{mm}$ waveguide length. This cavity raises several experimental challenges: (i) The probe transmission is intensity modulated by low-frequency random environmental fluctuations (e.g., temperature changes due to variations in the coupling ratio of the pump beam), which constantly shifts the FP cavity's transmission spectrum. In our experiments, this process generates a fluctuation of standard deviation $10^{5}$ times higher than the Brillouin signal. (ii) Intensity modulation of the pump signal modulates the cavity refractive index via the Kerr effect. The resulting time-dependent shift of the FP cavity transmission spectrum modulates the probe signal. Hence, pump intensity variations are transferred to the probe signal and generate a fluctuating background of standard deviation 2 times larger than the Brillouin peak gain. (iii) In our work, the chip is coupled via two 1-m-long standard single-mode fiber patchcords [53] in which the pump and probe counterpropagate and, as a result, stimulated Raman scattering (SRS) occurs along those fibers and generates a fluctuating background signal. This process contributes to a lesser extent to the system uncertainty $(\approx 10 \%$ of the Brillouin peak gain). Note that $\mathrm{SRS}$ in $\mathrm{Si}_{3} \mathrm{~N}_{4}$ is considered much smaller than for silica [15] and can be neglected [48].

To overcome the three aforementioned challenges, we developed a novel technique, triple intensity modulation (TIM), able to measure the exact Brillouin gain profile. This technique is detailed hereafter. The pump and probe beams are intensity modulated at frequency $f_{P}$ and $f_{S}$, respectively, and the detection is made at the frequency 


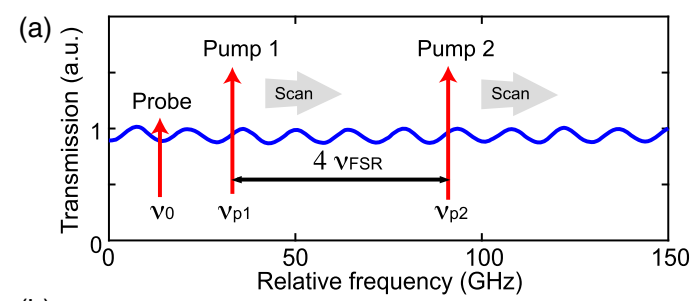

(b)

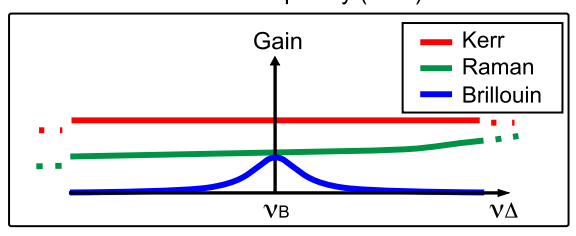

(c)

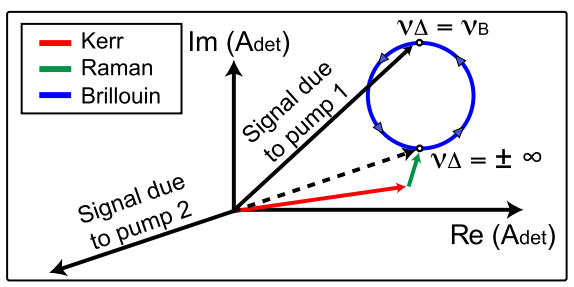

(d)

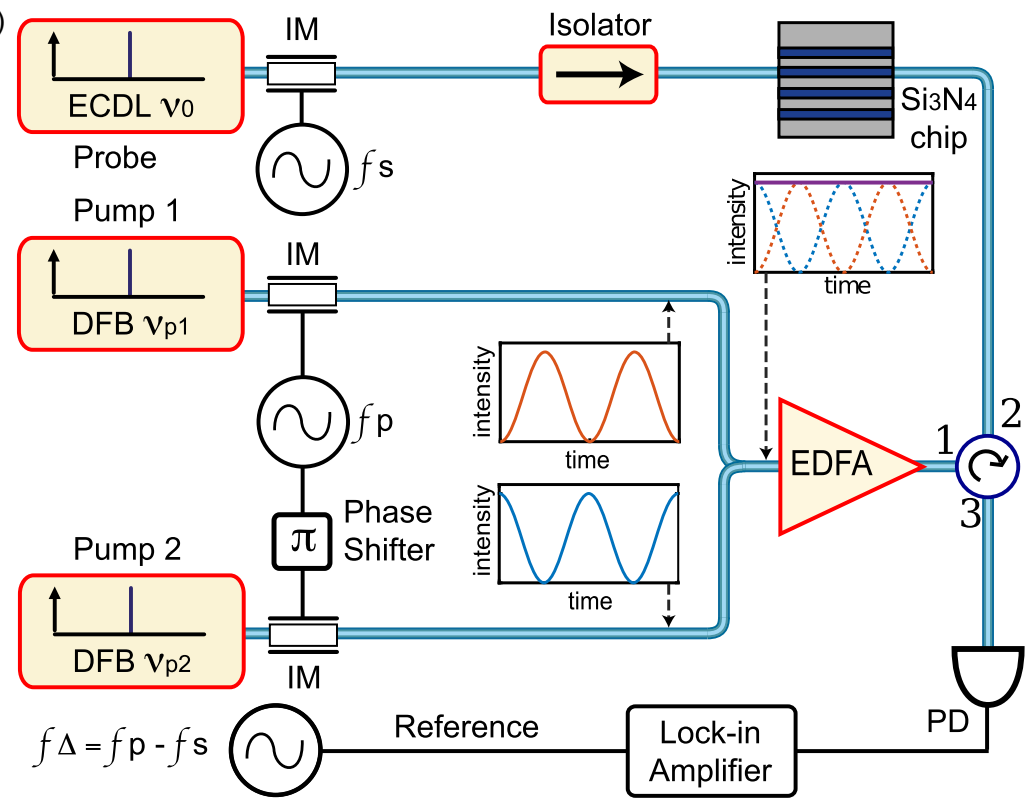

FIG. 2. High sensitivity SBS gain measurement scheme. (a) Measured waveguide transmission spectrum as a function of the relative frequency starting at $193.55 \mathrm{THz}$. (b) Illustration of the gains due to Kerr effect (phase-to-intensity conversion) and SRS as a function of frequency. (c) Illustration in the complex plane of different contributions of the measured probe signal amplitude $A_{\text {det }}$. The signal generated by pump 1 is a sum of the Kerr effect, SRS and SBS, while the signal generated by pump 2 only contains the Kerr effect and SRS. (d) Simplified TIM experimental setup. ECDL, external-cavity diode laser; DFB laser, distributed feedback laser; EDFA, erbium-doped fiber amplifier; IM, intensity modulator; PD, photodetector.

difference $f_{\Delta}=f_{P}-f_{S}$ (in our experiment, $f_{P} \approx f_{S}=$ $20 \mathrm{MHz}$ and $f_{\Delta}=75 \mathrm{kHz}$ ). This implementation takes advantage of the nonlinear nature of SBS leading to sumdifference frequency generation, and has two benefits: (i) It shifts the signal detection frequency away from the directcurrent (dc) frequency, where the low-frequency environmental noises lie. (ii) It efficiently filters the pump reflection out from the probe beam in the radio-frequency (rf) domain. The filtering is realized by detecting the signal at a distant frequency from the modulated pump frequency via a lock-in amplifier, eliminating the need for high extinction optical filtering. Such a technique is commonly used to filter out stray light in Brillouin microscopy [63].

The aforementioned Kerr effect and Raman scattering issues are resolved by canceling the temporal variation of the pump intensity. A similar method has been developed to cancel the nonresonant background in coherent anti-Stokes Raman spectroscopy [64]. This is realized by adding a second modulated pump beam (i.e., pump 2), whose intensity and polarization match perfectly those of the first pump (i.e., pump 1). The optical frequency of pump $2\left(\nu_{\mathrm{P} 2}\right)$ is increased compared to that of pump $1\left(\nu_{\mathrm{P} 1}\right)$ by a multiple of the chip FP cavity FSR: $\nu_{P 2}=\nu_{P 1}+n \nu_{\mathrm{FSR}}, n \in \mathbb{N}$ (in our experiment, $n=4$ ), as illustrated in Fig. 2(a). In this way, both pumps' intensities remain identical at all times inside the chip, irrespective of environmental fluctuations. In addition, the two pumps are intensity modulated at the same frequency $f_{P}$ but are $\pi$-phase shifted, such that perfect intensity cancellation of the modulation frequency
$f_{P}$ occurs. In this case, due to the constant total pump intensity, the Kerr effect no longer modulates the intensity of the output probe light, and hence will not generate any background noise. Moreover, the SRS gain experienced by the probe is nearly identical for each pump, because the frequency difference of the two pumps, $\nu_{P 2}-\nu_{P 1}$, is much smaller than the silica Raman gain bandwidth $(\sim 7 \mathrm{THz})$.

Since the two pumps are modulated with $\pi$-phase difference, SRS is attenuated at the detection frequency $f_{\Delta}=f_{P}-f_{S}$. For SBS, however, the probe only interacts with pump 1. The situation is summarized in Fig. 2(b) in which the gain response of the Kerr effect (phase-tointensity conversion can be assimilated to a gain process for the probe beam), SRS and SBS are sketched as a function of the pump-probe detuning frequency $\nu_{\Delta}=$ $\nu_{\mathrm{P} 1}-\nu_{0}$, where $\nu_{0}$ is the probe frequency. It can be seen that the Kerr effect is spectrally flat over the entire detuning frequency range and the Raman gain of the silica patchcord is nearly flat over the Brillouin gain bandwidth. Thus, these two contributions result in an identical gain for the two pumps. As the pumps show a $\pi$-phase difference, these effects cancel out at the detection frequency $f_{\Delta}$. A different view of this cancellation is represented in Fig. 2(c) where the different contributions of the probe detection signal $A_{\text {det }}$, are represented in the complex plane, for the two pumps. The Kerr effect and SRS generate a background signal (dotted arrow) that is canceled by pump 2 . The blue circle represents the path of the SBS Lorentzian amplitude line shape that is traced when the pump-probe detuning 
frequency $\nu_{\Delta}$, is scanned. The Brillouin gain spectral shape is measured by scanning the two pump frequencies $\nu_{P 1}$ and $\nu_{P 2}$, while fixing the probe frequency $\nu_{0}$. The two pump frequencies are scanned synchronously such that their frequency difference $\nu_{P 2}-\nu_{P 1}$ remains constant during the scanning, as illustrated in Fig. 2(a).

The experimental setup is shown in Fig. 2(d). The two pumps are generated by two distributed feedback (DFB) lasers and frequency scanning is performed by temperature control of these lasers [48]. In the small gain approximation [58], the magnitude of the measured amplitude $\left|A_{\text {det }}\right|$ can be written as [48]

$$
\left|A_{\mathrm{det}}\left(\nu_{\Delta}\right)\right| \propto \rho_{\mathrm{pd}} P_{S} P_{P} L \mathcal{L}\left(\nu_{\Delta}\right) g_{B} / A_{\mathrm{eff}},
$$

where $\rho_{\text {pd }}$ is the photodetector power-to-voltage conversion factor, $P_{S}$ is the time-averaged detected probe power, $P_{P}$ is the time-averaged pump power in the waveguide (pump 1 only), $g_{B}$ is the Brillouin peak gain, $L$ is the waveguide length, and $\mathcal{L}\left(\nu_{\Delta}\right)$ is the gain line shape.

Figure 3(a) shows the magnitude of the measured amplitude $\left|A_{\text {det }}\right|$, when the pump-probe detuning frequency is scanned from 10 to $35 \mathrm{GHz}$. The peak to the left is the silica Brillouin gain of the 1-m-long fiber patchcords connecting to the waveguide, and the signal around $25 \mathrm{GHz}$ is the $\mathrm{Si}_{3} \mathrm{~N}_{4}$ Brillouin gain. Since these two gains are measured jointly, their ratio provides an additional way to estimate the $\mathrm{Si}_{3} \mathrm{~N}_{4}$ Brillouin gain value. Figure 3(b) shows the measured
$\mathrm{Si}_{3} \mathrm{~N}_{4}$ gain spectrum along with our simulation results. A main peak reaching $(8 \pm 1) \times 10^{-14} \mathrm{~m} / \mathrm{W}$ is found at $25 \mathrm{GHz}$, accompanied by other smaller peaks [48]. Using the phase matching condition, the velocity of the main acoustic mode is calculated as $10.5 \mathrm{~km} / \mathrm{s}$, which agrees with the literature [65]. The full width at half maximum of the main peak $\Delta \nu=390 \mathrm{MHz}$, is obtained by a Lorentzian fitting. Figure 3(d) shows the FEM simulations performed over a 3D trench of the entire chip cross-section including the $\mathrm{SiO}_{2}$-air top interface and $\mathrm{SiO}_{2}$-silicon-substrate bottom interface. The simulation model is built using the precisely measured parameters from SEM [48], as shown in Fig. 3(c). Acoustic eigenmodes, computed from 21 to $27 \mathrm{GHz}$, show that the multiple peaks in the gain spectrum originate from the hybridization with high-overtone bulk acoustic resonances (HBARs) caused by the reflection of acoustic waves at the $\mathrm{SiO}_{2}$-air top interface and $\mathrm{SiO}_{2}$-silicon-substrate bottom interface [66].

Discussion.-In our $\mathrm{Si}_{3} \mathrm{~N}_{4}$ waveguide, the optical mode can be approximated as a transverse mode. Then, the Brillouin gain, $g_{B}$, can be expressed as [67]

$$
g_{B}=\frac{4 \pi^{2} n^{7} p_{12}^{2} \eta}{\lambda^{2} c \rho v_{a} \Gamma}
$$

where $n$ is the material refractive index, $c$ is the speed of light in vacuum, $\rho$ is the material density, $p_{12}$ is the photoelastic constant, $\Gamma$ is the acoustic damping and $\eta$ is the
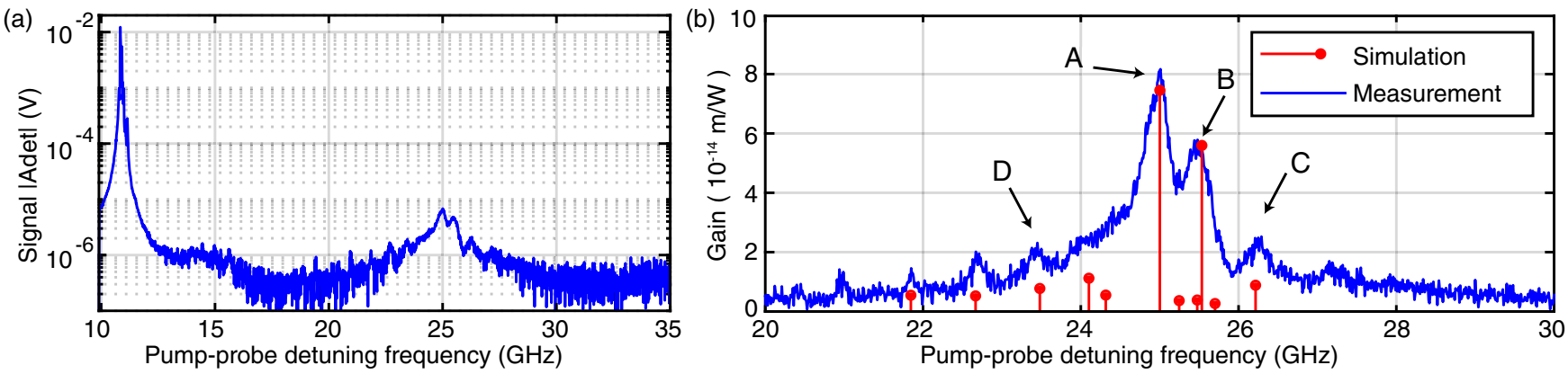

(c)

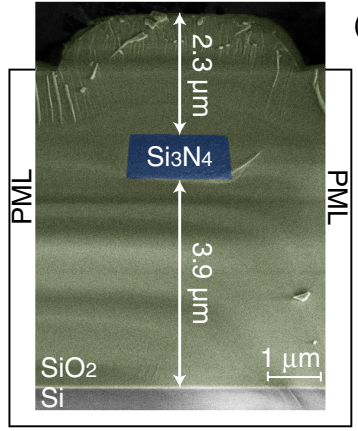

(d)

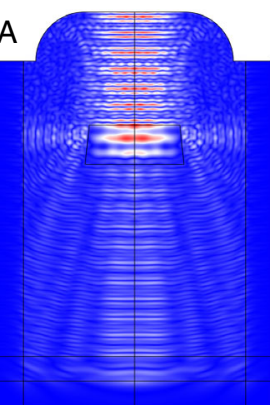

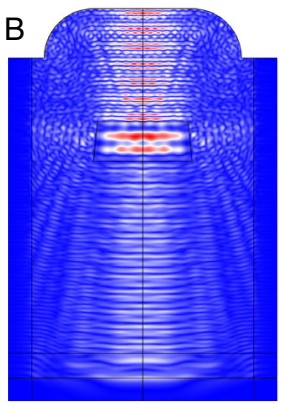


FIG. 3. Measurement and simulation results of $\mathrm{Si}_{3} \mathrm{~N}_{4}$ Brillouin gain spectrum. (a) Measured signal as a function of the pump-probe detuning frequency in logarithmic scale, showing the 1-m-long silica patchcord SBS to the left and $\mathrm{Si}_{3} \mathrm{~N}_{4} \mathrm{SBS}_{\text {at }} 25 \mathrm{GHz}$, with more than 3 orders of magnitude difference. (b) Measured $\mathrm{Si}_{3} \mathrm{~N}_{4} \mathrm{SBS}$ gain spectrum, in agreement with the simulated eigenmodes (eigenfrequency and gain for each eigenmode). (c) SEM image showing the sample cross section. The precisely measured geometry parameters are used to build the simulation model. (d) Normalized acoustic displacement field norm (instantaneous value) of four peaks extracted from the simulations. The top $\mathrm{SiO}_{2}$ dome-shaped interface is due to fabrication (see Supplemental Material S2 [48]). 
acousto-optic overlap coefficient. We now discuss on the three main parameters: $\Gamma, p_{12}$, and $\eta$.

The acoustic damping is proportional to the Brillouin linewidth as $\Gamma=\Gamma_{M}+\Gamma_{L}+\Gamma_{\text {inhomo }}=2 \pi \Delta \nu$, where $\Gamma_{M}$ represents material damping caused by phonon absorption, $\Gamma_{L}$ represents phonon leakage from the waveguide and $\Gamma_{\text {inhomo }}$ represents a linewidth broadening due to nonuniformity of the structure along the waveguide. The $\mathrm{Si}_{3} \mathrm{~N}_{4}$ Brillouin linewidth measured here is 10 times larger than that of silica, leading to a tenfold gain reduction. Our simulation gives a phonon leakage linewidth of $\Gamma_{L} / 2 \pi=41 \mathrm{MHz}$. According to the dimension tolerances given by the fabrication process, linewidth broadening caused by nonuniformity is computed to be $\Gamma_{\text {inhomo }} / 2 \pi \approx 28 \mathrm{MHz}$ [48]. Therefore, the remaining damping $(321 \mathrm{MHz})$ is assumed to be due to material absorption, in relative agreement with the expected material absorption following a frequencysquare law [68].

The photoelastic constant quantifies the amount of stress induced in the material by an electric field due to electrostriction. By fitting the peak heights of the FEM simulations over the measured gain spectrum, the magnitude of the photoelastic constant $\left|p_{12}\right|$ is estimated as [48]

$$
\left|p_{12}\right|=0.047 \pm 0.004
$$

This value corresponds to a 5.7 times reduction with respect to that of silica, leading to a 33 times gain decrease as $p_{12, \mathrm{Si}_{3} \mathrm{~N}_{4}}^{2}=p_{12, \mathrm{SiO}_{2}}^{2} / 33$.

The acousto-optic overlap coefficient is the coupling strength between the optical mode and the acoustic mode in relation to their spatial distributions. For example, almost perfect overlap of optical and acoustic modes is achieved in optical fibers, i.e., $\eta=1$. Our simulations show an acoustooptic overlap as $\eta_{\text {waveguide }} \approx 1 / 4$.

By substituting all these contributions into Eq. (2), we obtain a theoretical 342 times gain reduction in $\mathrm{Si}_{3} \mathrm{~N}_{4}$ waveguides compared to single-mode fibers. The Brillouin gain value measured in our $\mathrm{Si}_{3} \mathrm{~N}_{4}$ waveguide, in units of $\mathrm{m} / \mathrm{W}$, is 250 times smaller than the silica intrinsic gain, compatible with the theoretical value derived above. When the intrinsic gain is considered, however, both inhomogeneous broadening and phonon leakage are absent and acousto-optic overlap is unity $(\eta=1)$. Therefore, the intrinsic $\mathrm{Si}_{3} \mathrm{~N}_{4}$ Brillouin gain is estimated as $4 \times 10^{-13} \mathrm{~m} / \mathrm{W}, 51$ times smaller than that of silica.

Conclusion.-We have characterized backward SBS in integrated $\mathrm{Si}_{3} \mathrm{~N}_{4}$ waveguides. The observed $\mathrm{SBS}$ gain spectrum presents multiple peaks due to the hybridization with bulk acoustic resonance modes in the presence of $\mathrm{SiO}_{2}$ cladding of finite thickness. Note that the observed SBS frequency shift in $\mathrm{Si}_{3} \mathrm{~N}_{4}$ of $25 \mathrm{GHz}$ (corresponding to an acoustic velocity of $10.5 \mathrm{~km} / \mathrm{s}$ ) is the largest SBS frequency value reported on integrated platforms [16]. In addition, the SBS threshold [59] in our 5-mm-long waveguide is estimated as $87 \mathrm{~kW}$ [48]. To give two comparisons, a 1-m-long standard single-mode fiber has a SBS threshold of $100 \mathrm{~W}$ and a hypothetical silica waveguide with the same dimensions as our waveguide would have a SBS threshold power of $20 \mathrm{~kW}$. Since SBS usually limits the maximum optical power in waveguides, its high threshold in $\mathrm{Si}_{3} \mathrm{~N}_{4}$ proves the excellent high power handling capability of $\mathrm{Si}_{3} \mathrm{~N}_{4}$, central for integrated nonlinear photonics such as soliton microcomb [7] and chipbased supercontinuum generation [11].

The code and data used to produce the plots within this work will be released on the repository Zenodo upon publication of this Letter [69].

This work was supported by the Swiss National Science Foundation (SNSF) under Grant Agreement No. 159897, No. 178895, and No. 176563 (BRIDGE), and by funding from the European Union's H2020 research and innovation programme under Grant Agreement No. 732894 (FET Proactive HOT), and by the Defense Advanced Research Projects Agency (DARPA), Microsystems Technology Office (MTO) under Contract No. HR0011-15-C-0055 (DODOS). J.H. acknowledges the support provided by Professor Hwa-Yaw Tam and from the General Research Fund of the Hong Kong Government under project PolyU $152207 / 15 \mathrm{E}$. The $\mathrm{Si}_{3} \mathrm{~N}_{4}$ samples were fabricated in the EPFL center of MicroNanoTechnology (CMi).

*These authors contributed equally to this work. tobias.kippenberg@epfl.ch †luc.thevenaz@epfl.ch

[1] D. J. Moss, R. Morandotti, A. L. Gaeta, and M. Lipson, Nat. Photonics 7, 597 (2013).

[2] D. J. Blumenthal, R. Heideman, D. Geuzebroek, A. Leinse, and C. Roeloffzen, Proc. IEEE 106, 2209 (2018).

[3] M. A. Foster, A. C. Turner, J. E. Sharping, B. S. Schmidt, M. Lipson, and A. L. Gaeta, Nature (London) 441, 960 (2006).

[4] K. Luke, A. Dutt, C. B. Poitras, and M. Lipson, Opt. Express 21, 22829 (2013).

[5] M. H. P. Pfeiffer, C. Herkommer, J. Liu, T. Morais, M. Zervas, M. Geiselmann, and T. J. Kippenberg, IEEE J. Sel. Top. Quantum Electron. 24, 1 (2018).

[6] T. J. Kippenberg, S. M. Spillane, and K. J. Vahala, Phys. Rev. Lett. 93, 083904 (2004).

[7] T. J. Kippenberg, A. L. Gaeta, M. Lipson, and M. L. Gorodetsky, Science 361, eaan8083 (2018).

[8] B. Stern, X. Ji, Y. Okawachi, A. L. Gaeta, and M. Lipson, Nature (London) 562, 401 (2018).

[9] A. S. Raja, A. S. Voloshin, H. Guo, S. E. Agafonova, J. Liu, A. S. Gorodnitskiy, M. Karpov, N. G. Pavlov, E. Lucas, R. R. Galiev, A. E. Shitikov, J. D. Jost, M. L. Gorodetsky, and T. J. Kippenberg, Nat. Commun. 10, 680 (2019).

[10] J. Liu, E. Lucas, A. S. Raja, J. He, J. Riemensberger, R. N. Wang, M. Karpov, H. Guo, R. Bouchand, and T. J. Kippenberg, arXiv:1901.10372.

[11] A. L. Gaeta, M. Lipson, and T. J. Kippenberg, Nat. Photonics 13, 158 (2019). 
[12] H. Guo, W. Weng, J. Liu, F. Yang, W. Hansel, C. S. Brès, L. Thévenaz, R. Holzwarth, and T. J. Kippenberg, arXiv: 1908.00871.

[13] J. S. Levy, M. A. Foster, A. L. Gaeta, and M. Lipson, Opt. Express 19, 11415 (2011).

[14] X. Xue, F. Leo, Y. Xuan, J. A. Jaramillo-Villegas, P.-H. Wang, D. E. Leaird, M. Erkintalo, M. Qi, and A. M. Weiner, Light 6, e16253 (2017).

[15] M. Karpov, H. Guo, A. Kordts, V. Brasch, M. H. P. Pfeiffer, M. Zervas, M. Geiselmann, and T. J. Kippenberg, Phys. Rev. Lett. 116, 103902 (2016).

[16] B. J. Eggleton, C. G. Poulton, P. T. Rakich, M. J. Steel, and G. Bahl, Nat. Photonics 13, 664 (2019).

[17] A. H. Safavi-Naeini, D. V. Thourhout, R. Baets, and R. V. Laer, Optica 6, 213 (2019).

[18] G. S. Wiederhecker, P. Dainese, and T. P. Mayer Alegre, APL Photonics 4, 071101 (2019).

[19] D. R. Ponikvar and S. Ezekiel, Opt. Lett. 6, 398 (1981).

[20] M. S. Kang, A. Nazarkin, A. Brenn, and P. S. J. Russell, Nat. Phys. 5, 276 (2009).

[21] M. Pang, X. Jiang, W. He, G. K. L. Wong, G. Onishchukov, N. Y. Joly, G. Ahmed, C. R. Menyuk, and P. S. J. Russell, Optica 2, 339 (2015).

[22] J.-C. Beugnot, S. Lebrun, G. Pauliat, H. Maillotte, V. Laude, and T. Sylvestre, Nat. Commun. 5, 5242 (2014).

[23] O. Florez, P. F. Jarschel, Y. a. V. Espinel, C. M. B. Cordeiro, T. P. Mayer Alegre, G. S. Wiederhecker, and P. Dainese, Nat. Commun. 7, 11759 (2016).

[24] I. S. Grudinin, A. B. Matsko, and L. Maleki, Phys. Rev. Lett. 102, 043902 (2009).

[25] M. Tomes and T. Carmon, Phys. Rev. Lett. 102, 113601 (2009).

[26] H. Lee, T. Chen, J. Li, K. Y. Yang, S. Jeon, O. Painter, and K. J. Vahala, Nat. Photonics 6, 369 (2012).

[27] K. Y. Yang, D. Y. Oh, S. H. Lee, Q.-F. Yang, X. Yi, B. Shen, H. Wang, and K. Vahala, Nat. Photonics 12, 297 (2018).

[28] R. Pant, C. G. Poulton, D.-Y. Choi, H. Mcfarlane, S. Hile, E. Li, L. Thévenaz, B. Luther-Davies, S. J. Madden, and B. J. Eggleton, Opt. Express 19, 8285 (2011).

[29] B. Morrison, A. Casas-Bedoya, G. Ren, K. Vu, Y. Liu, A. Zarifi, T. G. Nguyen, D.-Y. Choi, D. Marpaung, S. J. Madden, A. Mitchell, and B. J. Eggleton, Optica 4, 847 (2017).

[30] H. Shin, W. Qiu, R. Jarecki, J. A. Cox, R. H. Olsson Iii, A. Starbuck, Z. Wang, and P. T. Rakich, Nat. Commun. 4, 1944 (2013).

[31] R. Van Laer, B. Kuyken, D. Van Thourhout, and R. Baets, Nat. Photonics 9, 199 (2015).

[32] Q. Liu, H. Li, and M. Li, Optica 6, 778 (2019).

[33] K. Y. Song, M. G. Herráez, and L. Thévenaz, Opt. Express 13, 82 (2005).

[34] Y. Okawachi, M. S. Bigelow, J. E. Sharping, Z. Zhu, A. Schweinsberg, D. J. Gauthier, R. W. Boyd, and A. L. Gaeta, Phys. Rev. Lett. 94, 153902 (2005).

[35] D. Marpaung, B. Morrison, M. Pagani, R. Pant, D.-Y. Choi, B. Luther-Davies, S. J. Madden, and B. J. Eggleton, Optica 2, 76 (2015).

[36] J. Li, X. Yi, H. Lee, S. A. Diddams, and K. J. Vahala, Science 345, 309 (2014).
[37] W. Loh, J. Becker, D. C. Cole, A. Coillet, F. N. Baynes, S. B. Papp, and S. A. Diddams, New J. Phys. 18, 045001 (2016).

[38] S. Gundavarapu, G. M. Brodnik, M. Puckett, T. Huffman, D. Bose, R. Behunin, J. Wu, T. Qiu, C. Pinho, N. Chauhan, J. Nohava, P. T. Rakich, K. D. Nelson, M. Salit, and D. J. Blumenthal, Nat. Photonics 13, 60 (2019).

[39] J. Li, M.-G. Suh, and K. Vahala, Optica 4, 346 (2017).

[40] M. S. Kang, A. Butsch, and P. S. J. Russell, Nat. Photonics 5, 549 (2011).

[41] D. B. Sohn, S. Kim, and G. Bahl, Nat. Photonics 12, 91 (2018).

[42] E. A. Kittlaus, N. T. Otterstrom, P. Kharel, S. Gertler, and P. T. Rakich, Nat. Photonics 12, 613 (2018).

[43] M. Pang, W. He, X. Jiang, and P. S. J. Russell, Nat. Photonics 10, 454 (2016).

[44] Y. Antman, A. Clain, Y. London, and A. Zadok, Optica 3, 510 (2016).

[45] R. G. Smith, Appl. Opt. 11, 2489 (1972).

[46] R. Dehghannasiri, A. A. Eftekhar, and A. Adibi, Phys. Rev. A 96, 053836 (2017).

[47] R. Dehghannasiri, A. A. Eftekhar, and A. Adibi, in 2017 IEEE Photonics Conference (IPC) (IEEE, New York, 2017), pp. 135-136, https://ieeexplore.ieee.org/document/8116038.

[48] See Supplemental Material S2 at http://link.aps.org/ supplemental/10.1103/PhysRevLett.124.013902 for noise sources estimates, which includes Refs. [49-51]; S2 for fabrication details, which includes Refs. [4,52-55]; S3 for simulations details, which includes Refs. [56,57]; S4 for the detailed experimental setup; S5 for the detailed gain calculation, which includes Ref. [58]; S6 for the threshold calculation, which includes Ref. [59].

[49] T. Herr, Solitons and dynamics of frequency comb formation in optical microresonators, ICMP, Technical Report, 2013, https://infoscience.epfl.ch/record/188349.

[50] B. E. A. Saleh and M. C. Teich, Fundamentals of Photonics, Wiley Series in Pure and Applied Optics, 2nd ed. (Wiley, New York, NY, 2007).

[51] R. H. Stolen, J. P. Gordon, W. Tomlinson, and H. A. Haus, J. Opt. Soc. Am. B 6, 1159 (1989).

[52] J. Liu, A. S. Raja, M. H. P. Pfeiffer, C. Herkommer, H. Guo, M. Zervas, M. Geiselmann, and T. J. Kippenberg, Opt. Lett. 43, 3200 (2018).

[53] A. S. Raja, J. Liu, N. Volet, R. N. Wang, J. He, E. Lucas, R. Bouchand, P. Morton, J. Bowers, and T. J. Kippenberg, arXiv:1906.03194.

[54] Y. Xuan, Y. Liu, L. T. Varghese, A. J. Metcalf, X. Xue, P.-H. Wang, K. Han, J. A. Jaramillo-Villegas, A. A. Noman, C. Wang, S. Kim, M. Teng, Y. J. Lee, B. Niu, L. Fan, J. Wang, D. E. Leaird, A. M. Weiner, and M. Qi, Optica 3, 1171 (2016).

[55] J. Liu, A. S. Raja, M. Karpov, B. Ghadiani, M. H. P. Pfeiffer, B. Du, N. J. Engelsen, H. Guo, M. Zervas, and T.J. Kippenberg, Optica 5, 1347 (2018).

[56] H. O. Pierson, Handbook of Chemical Vapor Deposition: Principles, Technology and Applications (William Andrew, Norwich, 1999), p. 281.

[57] W. Qiu, P. T. Rakich, H. Shin, H. Dong, M. Soljačić, and Z. Wang, Opt. Express 21, 31402 (2013). 
[58] R. W. Boyd, Nonlinear Optics (Elsevier, Burlington, 2008), pp. 436-440.

[59] R. W. Boyd, K. Rzazewski, and P. Narum, Phys. Rev. A 42, 5514 (1990).

[60] V. R. Almeida, R. R. Panepucci, and M. Lipson, Opt. Lett. 28, 1302 (2003).

[61] C. G. Poulton, R. Pant, and B. J. Eggleton, J. Opt. Soc. Am. B 30, 2657 (2013).

[62] P. Dainese, P. S. J. Russell, N. Joly, J. Knight, G. Wiederhecker, H. L. Fragnito, V. Laude, and A. Khelif, Nat. Phys. 2, 388 (2006).

[63] W. T. Grubbs and R. A. MacPhail, Rev. Sci. Instrum. 65, 34 (1994).
[64] G. Marowsky and G. Lüpke, Appl. Phys. B 51, 49 (1990).

[65] C. Wolff, R. Soref, C. Poulton, and B. Eggleton, Opt. Express 22, 30735 (2014).

[66] H. Chen, N. F. Opondo, B. Jiang, E. R. MacQuarrie, R. S. Daveau, S. A. Bhave, and G. D. Fuchs, Nano Lett. 19, 7021 (2019).

[67] P. T. Rakich, C. Reinke, R. Camacho, P. Davids, and Z. Wang, Phys. Rev. X 2, 011008 (2012).

[68] B. A. Auld, Acoustic Fields and Waves in Solids (Ripol Klassik, New York, 1973).

[69] https://dx.doi.org/10.5281/zenodo.3568423. 